\title{
Effects of naloxone on Morris water maze learning in the rat: Enhanced acquisition with pretraining but not posttraining administration
}

\author{
MICHAEL W. DECKER, INES B. INTROINI-COLLISON, and JAMES L. McGAUGH \\ University of California, Irvine, California
}

\begin{abstract}
Pretraining administration of the opiate antagonist naloxone on the first 4 of 5 days of training enhanced the acquisition of spatial information in the Morris water maze. Naloxone-treated rats learned to find an invisible escape platform located in a fixed position relative to environmental cues more rapidly than did control rats. Naloxone-treated rats also displayed better knowledge of the platform location during a probe trial conducted in the absence of the escape platform on Day 5 . In contrast, posttraining administration of naloxone did not significantly affect performance, even when a higher dose was used or when the training protocol was altered. These results suggest a role for endogenous opioid-peptidergic systems in the acquisition of spatial information.
\end{abstract}

Posttraining administration of opiate antagonists enhances the retention performance of rats and mice tested on a variety of learning and memory tasks, including inhibitory avoidance, active avoidance, habituation, brightness discrimination, and latent inhibition (for reviews, see Gallagher, 1985; McGaugh, 1989). The effect of opiate antagonists on spatial memory is unclear, however. Gailagher and her colleagues (Gallagher, Bostock, \& King, 1985; Gallagher, King, \& Young, 1983) found that posttraining opiate-antagonist treatment enhances the rate at which rats previously trained on an eight-arm radial maze attain criterion performance when the maze is placed in a novel spatial environment. These results would appear to suggest that endogenous opioid peptides are involved in the acquisition of spatial information. Gallagher and her colleagues, however, did not find a significant effect of posttraining administration of an opiate antagonist on initial acquisition of the eight-arm radial maze (Gallagher et al., 1985). Initial acquisition of the radial-maze task requires considerably more training than is the case in tasks typically used in assessing the effects of opiate blockade on learning and memory. Furthermore, the first few days involve familiarization with the task requirements as well as the acquisition of spatial information. Gallagher et al. (1985) suggested that this slower rate of acquisition may have been responsible for their failure to observe naloxone enhancement of initial acquisition of the radialmaze task.

The present experiments were designed to examine the role of opioid peptidergic systems in the initial acquisi-

The authors wish to thank T. Michael Gill and Ali Islami for technical assistance, and Nan Collett for help in the preparation of the manuscript. This work was supported by USPHS Postdoctoral Fellowship AG05446 (M.W.D.) and USPHS Grant MH12526 and Office of Naval Research Contract N00014-87-K-0518 (J.L.M.). Correspondence may be addressed to Michael Decker, Center for the Neurobiology of Learning and Memory, University of California, Irvine, CA 92717. tion of spatial information using the Morris water maze (Morris, 1981). We chose this task because rats learn the Morris water maze more quickly than they learn the radial-arm maze. In the water maze, animals learn to find a hidden escape platform in a large pool of water. Since no proximal cues are available and the use of odor trails is impossible, animals typically find the hidden platform by learning its location within the configuration of cues available in the testing environment. The search pattern used by rats during trials in which the escape platform is removed indicates that rats use spatial information in locating the platform. The role of endogenous opiates in modulating learning and memory has not, to this point, been examined in experiments using the Morris water maze. We now report that pretraining, but not posttraining, systemic administration of the opiate antagonist naloxone enhances the rate of acquisition of spatial information in this task.

\section{EXPERIMENT 1}

\begin{abstract}
Method
Subjects. Male Sprague-Dawley rats (Charles River) weighing 200-250 g were used in this study. The rats were singly housed and maintained on a 12:12 h light:dark cycle (lights on at 7:00 a.m.). Behavioral testing was conducted during the light portion of the cycle.

Behavioral Methods. The water maze used was a circular, galvanized-steel watering tank measuring $1.83 \mathrm{~m}$ in diameter and $0.58 \mathrm{~m}$ in height, filled to a depth of $20 \mathrm{~cm}$ with $25^{\circ} \mathrm{C}$ water. Attached to the rim of the tank along $1.70 \mathrm{~m}$ of its circumference was a white paper strip that extended down $16 \mathrm{~cm}$ from the rim. Other cues were also available in the environment surrounding the maze. Four starting positions equally spaced around the perimeter of the pool were used, dividing the tank into four equal quadrants. Located in the center of one of these quadrants was a $12 \times 14 \mathrm{~cm}$ rectangular clear Plexiglas platform $19 \mathrm{~cm}$ in height (i.e., its surface was $1 \mathrm{~cm}$ below water level). This platform remained in a fixed location throughout testing.
\end{abstract}


Each trial began with the rat being placed in the water, facing the wall of the tank, at one of the four start positions. The start positions were varied quasi-randomly across trials with the constraint that each block of two trials use one start position near the platform and one farther away. The rat was allowed to swim until it located and climbed onto the escape platform. If the rat did not locate the platform within $90 \mathrm{sec}$, it was gently guided to it. The rat remained on the platform for $20 \mathrm{sec}$ before being removed. The second trial in each block was conducted after a delay of 5-10 sec.

The rats in Experiment 1 received two trials per day for 5 days. Intraperitoneal injections of naloxone $(0,1.0$, or $3.0 \mathrm{mg} / \mathrm{kg})$ were administered either 5 min before or immediately after each session on Days 1 through 4 , yielding a total of six groups ( $n=9 /$ group). No injections were made on Day 5 . Following the last trial on Day 5, a free-swim probe trial was conducted. For this probe trial, the rat was allowed to swim for $60 \mathrm{sec}$ in the maze with the escape platform removed. The rat's behavior during the probe session was recorded on videotape using an overhead camera. Using this videotape record of the free swim, the amount of time each animal spent in the target quadrant (the quadrant that had contained the escape platform during training) was determined. We also recorded the number of times the rat crossed the place where the platform had been located during training as well as the number of times the rat crossed the equivalent portion of each of the other quadrants.

Drugs. Naloxone $\mathrm{HCl}$ (Sigma) was dissolved in $0.9 \%$ saline and injected in a volume of $1.0 \mathrm{ml} / \mathrm{kg}$. Doses were determined using the weight of the salt. Control animals received injections of saline.
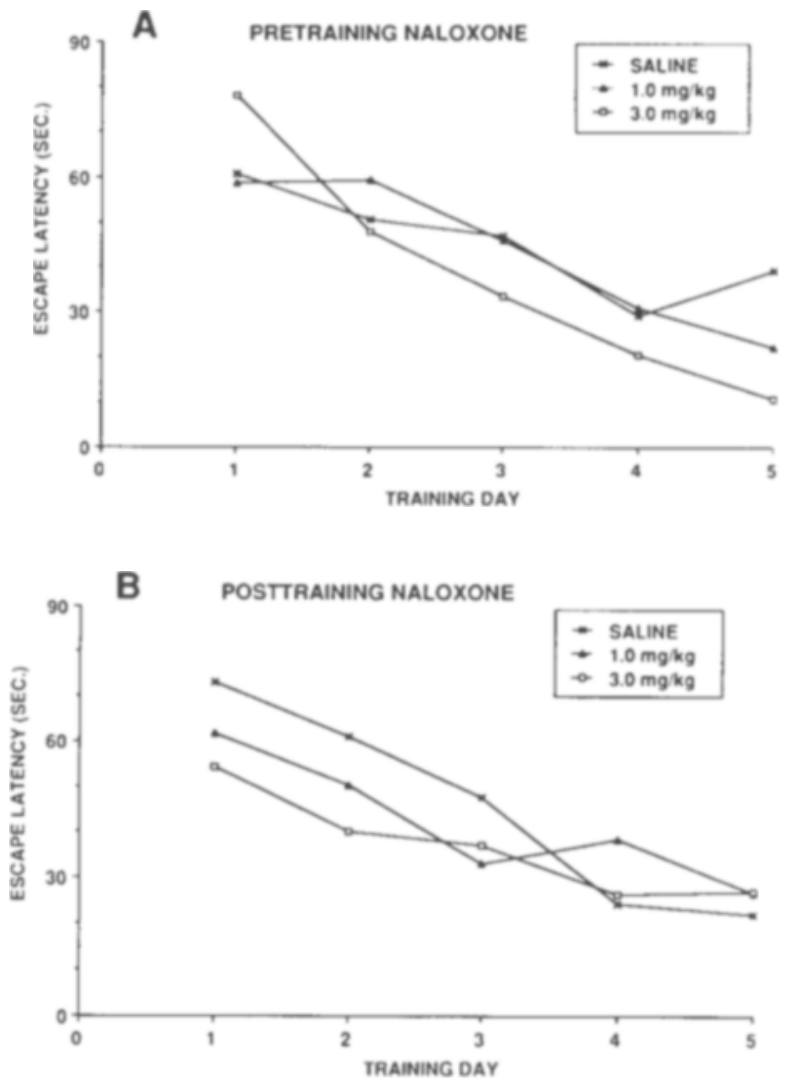

Figure 1. Effects of pretraining (A) and posttraining (B) administration of naloxone on escape latencies during training on the Morris water maze in Experiment 1. Escape latencies represented are the mean of two trials run on each day. Pretraining administration $(3.0 \mathrm{mg} / \mathrm{kg})$ enhanced the rate of decrease in escape latencies across days, whereas posttraining administration had no statistically significant effects. See text for statistical analysis.

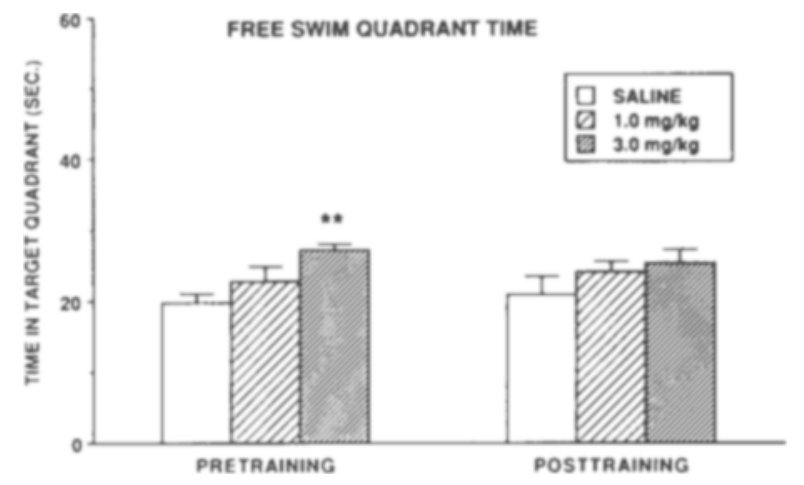

Figure 2. Time spent in the target quadrant during the 60-sec free swim conducted on Day 5 of training in Experiment 1. No drug was administered on this day. Rats given pretraining injections $(3.0 \mathrm{mg} / \mathrm{kg}$ ) of naloxone on Days $1-4$ spent significantly more time in the target quadrant than did control rats $\left({ }^{* *} p<.01\right)$. No statistically significant effects of posttraining administration of naloxone were noted.

\section{Results and Discussion}

The escape latencies of animals receiving pretraining injections are illustrated in Figure 1A. Latencies were significantly reduced as a function of training $[F(4,96)=$ $29.09, p<.0001$, for training days] and no overall drug effect was found $[F(2,24)=0.618, p>.50]$. However, pretraining injections of naloxone produced a clear dosedependent enhancement of the rate at which escape latencies decreased during training. This enhanced rate of acquisition is reflected in a statistically significant dose $x$ training interaction $[F(8,96)=2.92, p<.01]$. The escape latencies for animals receiving posttraining drug injections are presented in Figure 1B. Again, there was a significant effect of training $[F(4,96)=20.09, p<.0001$, for training days] but, in contrast to the effects of pretraining naloxone administration, posttraining injections of naloxone had no significant effect on escape latencies $[F(2,24)=1.83, p>.15$, for the main effect of drug; $F(8,96)=1.66, p>.1$, for the drug dose $\times$ training interaction].

The performance of the rats during the free-swim probe trial is represented in Figure 2. These results are consistent with the effects of naloxone on escape latencies during training. During this free swim, the rats that had received pretraining injections of naloxone on the previous 4 days spent more time in the training quadrant than did control rats $[F(2,24)=5.46, p<.02]$. Furthermore, as shown in Figure $3 \mathrm{~A}$, naloxone-treated rats crossed the actual location that had been occupied by the platform more frequently (relative to equivalent portions of the other quadrants) than did control rats $[F(2,24)=3.99$, $p<.05$, for the main effect of drug on total number of crossings; $F(3,72)=32.62, p<.0001$, for location of crossings; and $F(6,72)=2.26, p<.05$, for the interaction]. Although trends were found for both the 1.0- and the $3.0-\mathrm{mg} / \mathrm{kg}$ doses, the effects were statistically significant only at the higher dose. Rats receiving the $3.0-\mathrm{mg} / \mathrm{kg}$ posttraining dose of naloxone also tended to spend more time in the target quadrant than did control rats (Figure 2), 

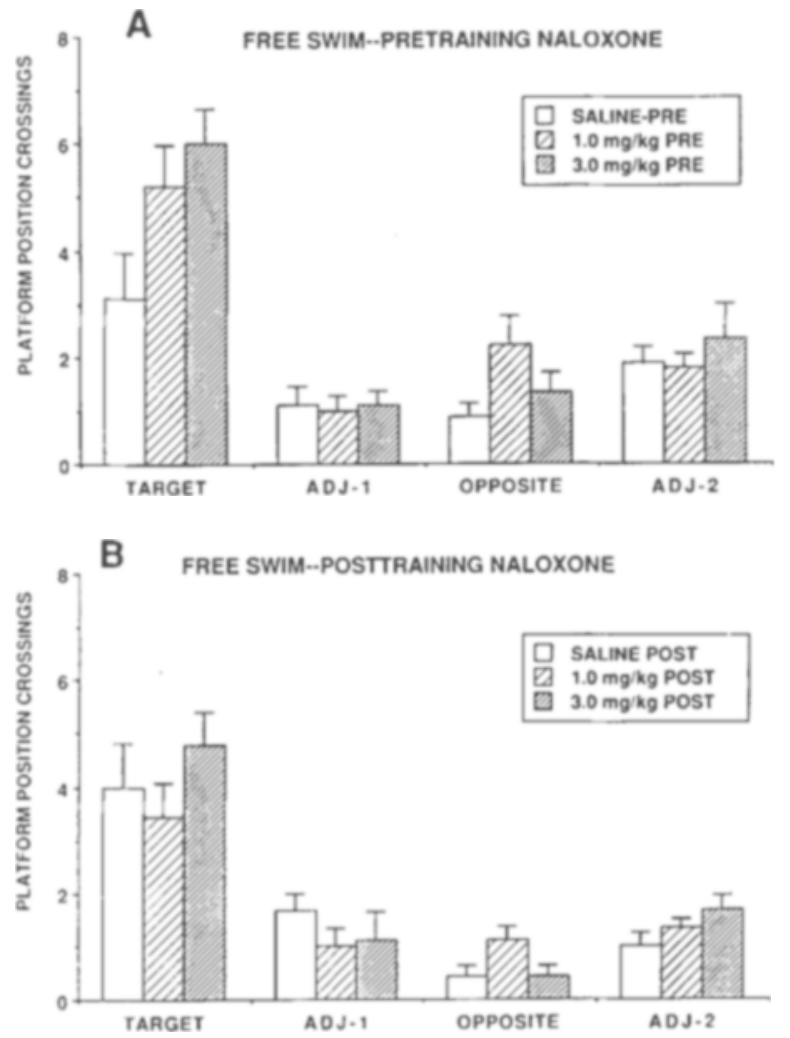

Figure 3. Number of platform-location crossings in each of the quadrants during the free swim on Day 5 for rats receiving pretraining (A) or posttraining (B) naloxone administration during Training Days 1-4 (Experiment 1). Rats given pretraining injections of naloxone $(3.0 \mathrm{mg} / \mathrm{kg}$ ) crossed the target platform location more frequently (relative to the other possible locations) than did control rats. Posttraining naloxone administration did not affect performance on this measure. See text for statistical analysis.

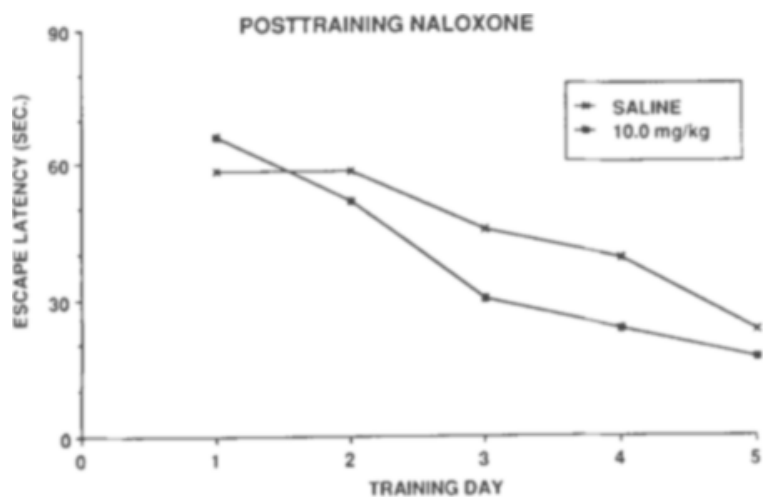

Figure 4. Effects of posttraining administration of naloxone (10.0 mg/kg) on escape latencies during training on the Morris water maze in Experiment 2. Escape latencies represented are the mean of two trials run on each day. No statistically significant differences were noted between groups. See text for statistical analysis.

but this difference was not statistically significant $[F(2,24)=$ $1.35, p>.25]$. Posttraining injections of naloxone in this range of doses also did not produce platform-location crossing patterns significantly different from those of control rats during the free-swim probe trial (Figure 3B)
$[F(2,24)=0.479, p>.60$, for dose effect; $F(3,72)=$ $36.07, p<.0001$, for location; and $F(6,72)=1.29$, $p>.25$, for the interaction].

Thus, whereas pretraining injection of naloxone enhanced performance on the water maze, posttraining injection in the same dose range did not significantly alter performance.

\section{EXPERIMENT 2}

Because the results with posttraining injection of the $3.0-\mathrm{mg} / \mathrm{kg}$ dose of naloxone suggested a trend toward improved performance in the free-swim probe trial in Experiment 1 (see Figure 2), we conducted an additional experiment using a posttraining injection of a higher dose of naloxone $(10.0 \mathrm{mg} / \mathrm{kg})$. The training procedures were the same as in Experiment 1 except that only the posttraining administration of naloxone was used $(0$ or $10.0 \mathrm{mg} / \mathrm{kg}$; $n=9$ (group) .

\section{Results and Discussion}

The results of this experiment can be seen in Figures 4 and 5. Latencies decreased with training experience $[F(4,64)=12.82, p<.0001]$; but although naloxonetreated rats displayed somewhat better performance than
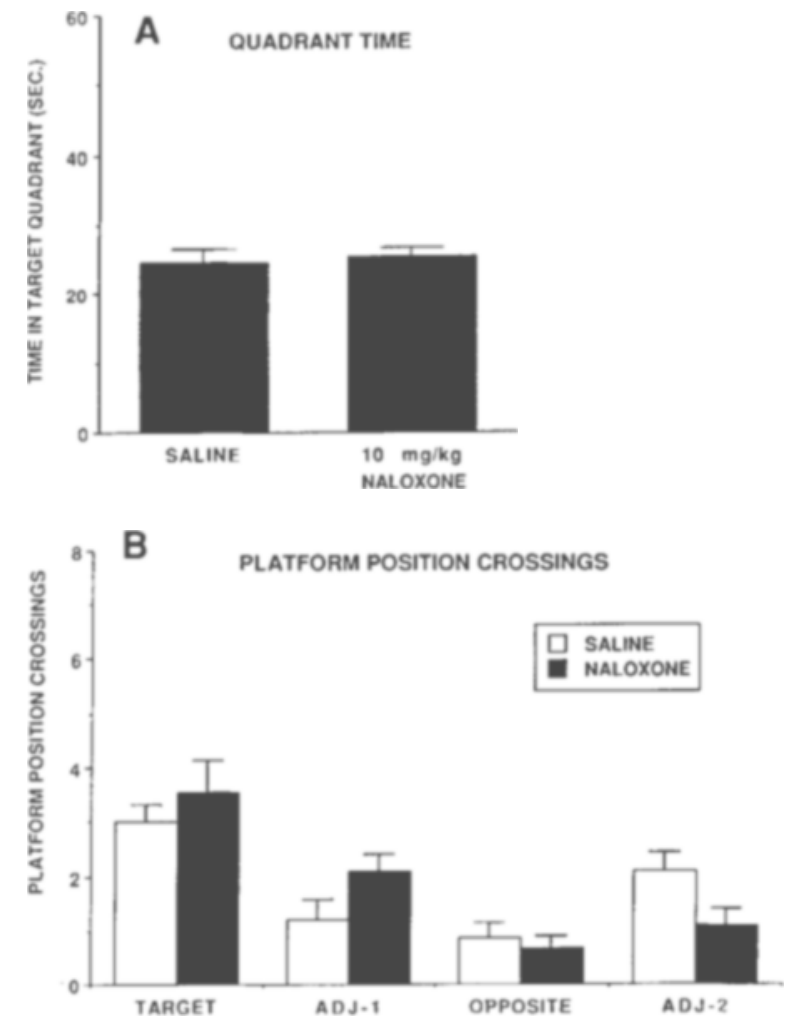

Figure 5. Time spent in the target quadrant (A) and number of platform-location crossings in each quadrant (B) during the 60-sec free swim conducted on Day 5 of training in Experiment 2. No drug was administered on this day. Rats given posttraining injections $(10.0 \mathrm{mg} / \mathrm{kg})$ of naloxone on Days $1-4$ did not differ significantly from control rats on either of these measures. See text for statistical analysis. 
did control rats on the escape-latency measure during training (see Figure 4), this effect was not statistically significant $[F(1,16)=1.98, p>.15$, for the drug main effect; $F(4,64)=0.97, p>.40$, for the drug $\times$ training interaction]. Furthermore, no statistically significant differences between these groups were noted during the free-swim probe trial on either the quadrant-time measure $[F(1,16)=0.142, p>.70]$ or the platform-position crossing measure $[F(1,16)=1.03, p>.30$, for drug effect; $F(3,48)=20.69, p<.0001$, for location; and $F(3,48)=1.22, p>.30$, for the interaction; Figure 5].

Thus, despite the apparent trend toward enhanced performance observed with posttraining administration of the higher dose of naloxone in Experiment 1, posttraining administration of a still higher dose did not significantly alter the rate of acquisition of spatial information. It is still possible, of course, that a dose between these two doses may yet prove effective.

\section{EXPERIMENT 3}

The results of our first two experiments demonstrated that although pretraining administration of naloxone enhanced the performance of rats in the water maze, posttraining injections were without effect. Generally, previous work demonstrating memory enhancement with naloxone has made use of the posttraining injection procedure (Gallagher, 1985; McGaugh, 1989), so it is perhaps surprising that in the present case only pretraining injections were effective. However, in the experiments by Gallagher and her colleagues (Gallagher et al., 1985, 1983 ), in which posttraining naloxone administration enhanced the rate of acquisition of new spatial information in rats trained on the radial-arm maze, a modified posttraining injection procedure was actually used. In those experiments, rats received drug injections after visiting four of the eight baited arms of the maze. The second portion of the trial was conducted some hours later. During this second part of the trial, the rats were allowed to retrieve the food pellets they had not obtained in the first part of the trial. This second part of the trial was not followed by drug administration.

Because acquisition of the new spatial information undoubtedly occurred during both portions of the day's trial, it is possible that the drug may have exerted its effect in these radial-arm maze experiments by altering the rat's acquisition of spatial information during the second half of the trial and not the first, as the description of this as a posttraining procedure implies. The finding of Gallagher et al. (1983) that injections made $2 \mathrm{~h}$ after the first part of the trial did not alter performance argues against the interpretation that naloxone has a direct proactive effect on the second half of the trial. It is possible, though, that some interaction between performance of the task and the immediately subsequent injection of naloxone enhanced the rats' ability to acquire spatial information during the second half of the trial.
In Experiment 3, we tested the possibility that a modification of the posttraining administration procedure similar to that used by Gallagher and her colleagues is necessary to observe naloxone-induced enhancement in spatial tasks. In this experiment, we assessed the effects of naloxone on the acquisition of spatial information in the water maze using an adaptation of the injection/training procedures used by Gallagher and her colleagues in their radialarm maze experiments described above.

\section{Method}

The apparatus and general training procedures were the same as in the first two experiments. In this experiment, however, we trained rats using two, two-trial training blocks per day. These blocks were separated by a 4-h delay, and injections of naloxone $(0,1.0,3.0$, or $10.0 \mathrm{mg} / \mathrm{kg} ; n=9 /$ group) were made immediately after the first training block (i.e., $4 \mathrm{~h}$ before the second training block). No injections were made following the second block on each day. This procedure was followed for 3 days. On the fourth day, the rats received only a $60-\mathrm{sec}$ free-swim probe trial.

\section{Results and Discussion}

The results of this experiment are found in Figures 6 and 7. Escape latencies decreased as a function of training $[F(5,160)=54.83, p<.0001$, for training blocks $]$, but administration of naloxone did not alter acquisition $[F(3,32)=1.82, p>.15$, for the drug effect; $F(15,160)=$ $1.146, p>.30$, for the drug $\times$ training interaction; Figure 6]. In addition, drug administration during training did not affect performance during the free-swim probe trial $[F(3,32)=2.01, p>.10$, for the drug effect on quadrant time; $F(3,32)=1.75, p>.15$, for the drug effect on number of crossings; $F(3,96)=13.16, p<$ .0001 , for location of crossings; and $F(9,96)=1.35$, $p>.20$, for the drug $\times$ location interaction; Figure 7].

Thus, posttraining administration of naloxone did not alter the rate of acquisition of spatial information in the water maze when we used a modified injection/training protocol based on that used by Gallagher and her colleagues. There are, of course, still several differences be-

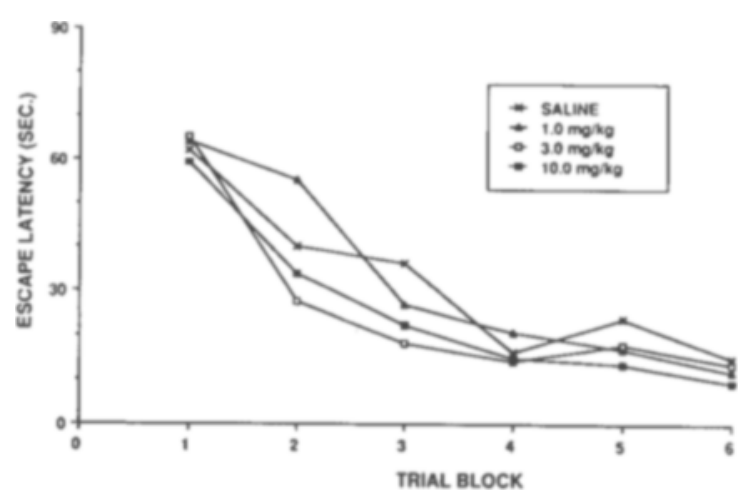

Figure 6. Escape latencies of rats trained using two, two-trial blocks per day in Experiment 3. Rats received naloxone immediately after the completion of the first block run on each day. The second training block on each day was conducted $4 \mathrm{~h}$ later. No statistically significant effects were noted (see text). 

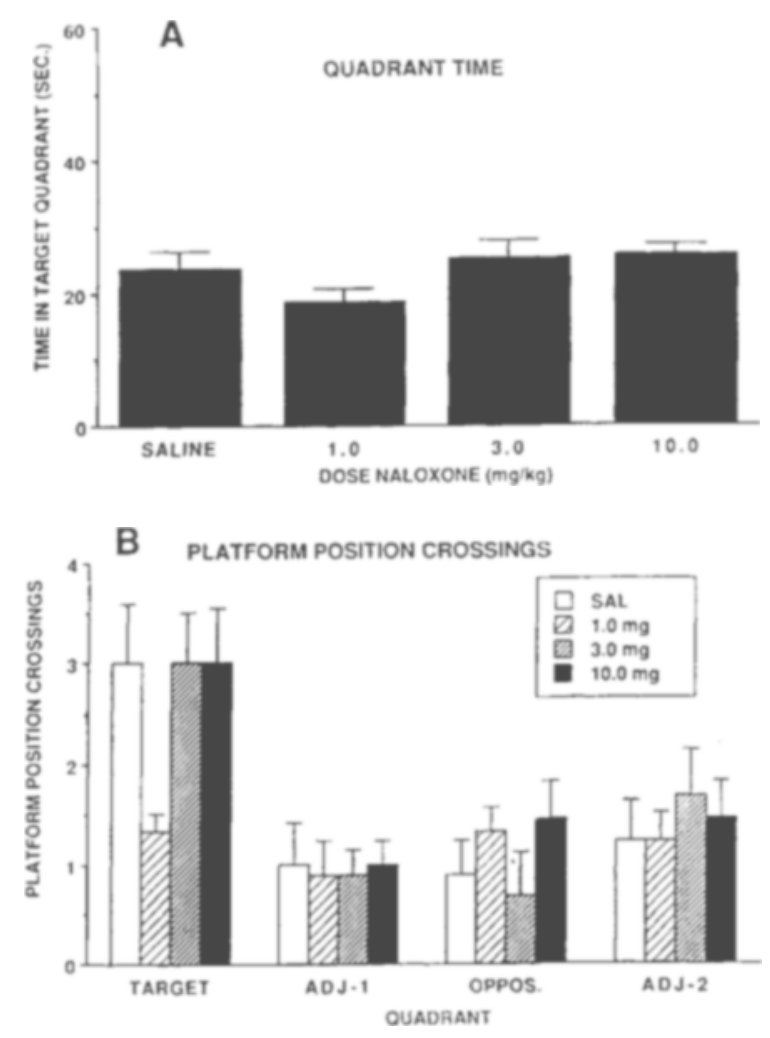

Figure 7. Amount of time spent in the target quadrant (A) and number of platform-location crossings in each of the quadrants (B) during the 60-sec free swim conducted on the day after the last training trial in Experiment 3. No statistically significant effects were noted on either measure. See text for statistical analysis.

tween our task and the radial-maze task used by Gallagher and her colleagues. Clearly, there are differences in the performance requirements and motivational factors involved. More importantly, we assessed the initial acquisition of a reference-memory spatial task, whereas Gallagher and her colleagues measured the acquisition of new spatial information in well-trained animals using a task that measures both working and reference memory.

\section{GENERAL DISCUSSION}

Our findings indicate that pretraining injections of the opiate antagonist naloxone improved acquisition on the Morris water maze. To our knowledge, pharmacological enhancement of the performance of normal adult rats has not previously been reported for this task. Although posttraining injections of naloxone were not effective, the effects of pretraining naloxone do not depend on the presence of the drug during testing: enhanced performance was seen on free-swim probe trials conducted on days when no drug was injected. Although effects on nonassociative factors influencing performance can never be completely discounted when pretraining injections are used, the increased "spatial bias" exhibited by rats on this drug-free probe trial suggests that the animals found the platform more rapidly during training because they had acquired superior knowledge regarding its location.

Interestingly, pretraining naloxone treatment did not immediately result in improved performance. Naloxone pretreatment appeared to increase escape latencies on the first day of training (see Figure 1). Although this effect is of only borderline significance in the current experiment $(p<.10)$, we consistently observed this tendency in pilot studies. A finding that pretraining naloxone administration impairs performance on the first day would be consistent with previous reports of the impairing effects of pretraining naloxone administration on active avoidance and habituation (Izquierdo, 1980). A more recent study has also demonstrated an impairment of the retention of autoshaping with pretraining naloxone administration (Messing, Allen, Aanonsen, \& Sparber, 1989). Our results, of course, differ from both of these in that we observed a naloxone-induced enhancement following additional training under the drug. This late development of the naloxone-induced enhancement in the current study is consistent with the view that this effect is due to enhanced acquisition of spatial information. Decreases in latencies on early trials in the Morris water maze probably do not reflect the acquisition of spatial information as much as they reflect the initiation of an active search strategy: rats trained to a platform moved for each trial escape as quickly as rats trained to a fixed location during the first few trials (Decker \& Brioni, 1988; Mactutus \& Murray, 1986). Thus, a treatment affecting the acquisition of spatial information would not necessarily be expected to be effective during these early trials. In contrast, this failure to observe reduced latencies among naloxone-treated rats during early trials would not be expected if the drug primarily affected nonassociative factors.

Despite our evidence for enhancing effects of pretraining administration of naloxone, posttraining treatment in this same dose range did not significantly affect performance. Further investigation of the posttraining effects of naloxone using a higher dose of the drug and a modification of the training procedure similarly failed to produce a significant effect on the acquisition of this task. Although it is still possible that additional modification of the task or the use of different doses might reveal an enhancing effect of naloxone administered posttraining on this task, the current findings contrast with several reports of memory enhancement obtained with posttraining opiateantagonist treatment using a variety of other learning and memory tasks (Gallagher, 1985; McGaugh, 1989). It is entirely possible, of course, that posttraining effects of naloxone are task-dependent. In this regard, it should be noted that the water maze differs considerably from most of the learning and memory tasks previously used to study the effects of opiate antagonists. Not only do most of the 
tasks previously used make use of nonspatial information, but these tasks typically require only a single training session. With respect to spatial-memory tasks, our findings with posttraining naloxone administration are consistent with those previously reported by Gallagher and her colleagues. As discussed earlier, Gallagher et al. (1985, 1983) found naloxone enhancement of the acquisition of new spatial information in rats previously trained on the radial-arm maze. They did not, however, find any effect of naloxone on initial acquisition of this task (Gallagher et al., 1985)-a finding consistent with our failure to find a posttraining effect in the water maze.

Our results suggest that the rate of acquisition of spatial information during initial training in the Morris water maze can be enhanced by pretraining administration of naloxone, an opiate antagonist. This finding, coupled with the reports by Gallagher and her colleagues that naloxone enhances the rate of acquisition of new spatial information in rats previously trained on the radial-arm maze, suggests that endogenous opioid peptides play a role in the learning of spatial information. The failure of posttraining administration of naloxone to affect initial acquisition of the water maze task in the current study, and of the radial-arm maze in the Gallagher et al. (1985) study, however, suggests that further work will be necessary to fully elucidate that role.

\section{REFERENCES}

Decker, M., \& Brioni, J. (1988). |Effects of training to a fixed vs. a randomly placed platform in the Morris water maze|. Unpublished raw data.

Gallagher, M. (1985). Re-viewing modulation of learning and memory. In N. M. Weinberger, J. L. McGaugh, \& G. Lynch (Eds.), Memory systems of the brain: Animal and human cognitive processes (pp. 311-334). New York: Guilford.

Gallagher, M., Bostock, E., \& King, R. (1985). Effects of opiate antagonists on spatial memory in young and aged rats. Behavioral \& Neural Biology, 44, 374-385.

Gallagher, M., King, R. A., \& Young, N. B. (1983). Opiate antagonists improve spatial memory. Science, 221, 975-976.

IzQUiERDO, I. (1980). Effects of a low and high dose of $\beta$-endorphin on acquisition and retention in the rat. Behavioral \& Neural Biology, 30, $460-464$.

Mactutus, C. F., \& Murray, D. L. (1986). Spatial memory: Winstay vs. win-shift strategies. Society for Neuroscience Abstracts, 12 , 520 .

McGaugh, J. L. (1989). Involvement of hormonal and neuromodulatory systems in the regulation of memory storage. Annual Review of Neuroscience, 12, 255-287.

Messing, R. B., Allen, S. J., annonsen, L., \& Sparber, S. G. (1989). Naloxone administration impairs autoshaped learning. $B e-$ havioral \& Neural Biology, 51, 34-45.

Morris, R. G. M. (1981). Spatial localization does not require the presence of local cues. Learning \& Motivation, 12, 239-260.

(Manuscript received January 20, 1989; revision accepted for publication April 17, 1989.) 